\title{
FSO system performance analysis based on novel Gamma-Chi-square irradiance PDF model
}

\author{
Jelena TODOROVIĆ ${ }^{1 *}$, Petar SPALEViĆ ${ }^{1}$, Stefan PANiĆ ${ }^{2 *}$, \\ BOJANA MILOSAVLJEVIĆ ${ }^{3}$, MiLAN GLIGORIJEVIĆ ${ }^{4}$ \\ ${ }^{1}$ Faculty of Technical Sciences, University of Priština - Kosovska Mitrovica, \\ Knjaza Miloša 7, 38220 Kosovska Mitrovica, Serbia \\ ${ }^{2}$ Faculty of Sciences, University of Priština - Kosovska Mitrovica, Faculty of Sciences, \\ Lole Ribara 29, 38220 Kosovska Mitrovica, Serbia \\ ${ }^{3}$ Higher Technical Professional School in Zvečan, \\ Nušićeva 6, 38227 Zvečan, Serbia \\ ${ }^{4}$ Academy of Criminalistics and Police Studies, \\ University of Criminal Investigation and Police Studies, \\ Cara Dušana 196, 11080 Zemun, Serbia \\ *Corresponding authors: Jelena Todorović-jelena.todorovic@pr.ac.rs, Stefan Panić-stefan.panic@pr.ac.rs
}

In order to provide a novel analytically traceable free space optics (FSO) channel model for describing turbulence based irradiance fluctuations, following basic scintillation theory principles, we have derived closed-form expression for probability density function (PDF) of a new statistical Gamma-Chi-square model. Further, capitalizing on provided model, error performances of FSO system over on-off keying (OOK) transmission scheme both in the presence of atmospheric turbulence and misalignment fading (pointing error) is investigated. For both cases, the average bit error rate (ABER) at the receiving side of the system is determined in an analytically closed form. The results are graphically presented in order to analyze the impact of different levels of turbulence, as well as other relevant parameters, on the quality of the received signal in the OOK modulated FSO system.

Keywords: free space optical (FSO), Gamma-Chi-square distribution, atmospheric turbulence, pointing error, on-off keying (OOK).

\section{Introduction}

One of the main issues in the process of analyzing transmission aspects of free space optics (FSO) is introducing analytically tractable mathematical model for probability 
density function (PDF) of randomly variable irradiance signal fluctuating under atmospheric induced turbulence. Up to now many various channel distribution models have been proposed for modeling different levels of atmospheric turbulence influence: Lognormal-Rician model [1], $K$ model [2],$I-K$ model [3] , Gamma-Gamma model [4], Malaga model [్], double Weibull model []], double generalized Gamma model [7]]. The double generalized Gamma distribution, includes important distributions such as Gamma-Gamma, double Nakagami, double Weibull, and double Rayleigh, and it can also efficiently describe the Nakagami/Rayleigh-Lognormal as well as Gamma-Gamma [8]. For all these models it has been shown that they fit obtained measurement results in a wide range of turbulence conditions, since they are all formed by simultaneous influence of both independent large-scale and small-scale turbulence eddy effects. In $[\underline{9}, \underline{10}]$ it has been explained how Lognormal-Rician excellently matches simulated and experimentally obtained data. However, in [10] PDF and CDF of Lognormal -Rician model they are approximated, since their forms are not analytically traceable in closed-forms. In [11-16] it has been shown how Rician (Chi-square) model can efficiently model turbulence eddy effects in a wide range of conditions.

Motivated by the issues stated above, in this paper we will propose a novel analytically traceable Gamma-Chi-square (Gamma-Rician) PDF model, obtained as a product of Gamma model and Chi-square (Rician) model. Based on results and analysis $[\underline{4}, \underline{10}, \underline{11}, \underline{17}, \underline{18}]$, Gamma model excellently represents a small-scale, while Chi-square (Rician) model represents large-scale turbulence eddy effects. Further, we will generalize our PDF model, by taking into account performance deteriorating effects of misalignment-induced fading, through introducing a boresight pointing error model into newly obtained Gamma-Chi-square distribution, similarly as these effects were taken into account in [19-22]. Closed-form PDF expressions will be derived for such FSO channel model, which is another contribution of this manuscript. Finally, in order to carry out a comprehensive performance analysis of FSO transmission with on-off keying (OOK) over our newly introduced channel model, we will observe standard performance criterion of average bit error rate (ABER) and will analyze it in the function of transmission system parameters. For both cases, in the presence of atmospheric turbulence with and without misalignment fading, the ABER expressions at the receiving side of the system are determined in an analytically closed form. Our performance analysis can be seen as a generalization of the analysis provided in [22-24].

The following structure of the manuscript is organized in several sections. In Section 2, the obtained PDF expressions for analyzed system models are given, both for atmospheric turbulence and pointing error case. In Section 3, initial and analytical close form expressions for the ABER for Gamma-Chi-square distribution in both mentioned cases are derived and presented. The obtained numerical results are presented and discussed in Section 4, for different levels of atmospheric turbulence and different values of relevant parameters. Obtained results have been analytically verified through Monte Carlo simulations. Section 5, summarizes the conclusions. 


\section{System and channel model}

\subsection{Atmospheric turbulence model}

As previously stated, a well-known approach in the scintillation theory [4] is to express the irradiance of the received optical wave as the product of two irradiances occurring from small and large scale turbulent eddies, i.e. $I_{\mathrm{a}}=I_{x} I_{y}$, with assumption that $I_{x}, I_{y}$ are statistically independent random processes. The signal is mostly affected by random variations in the refractive index across the different atmospheric eddies, which are a consequence of turbulence. During the optical signal transmission through the atmospheric channel, the laser beam and eddies interact, which results in phase and amplitude fluctuations of the received optical signal $[\underline{4}, \underline{5}, 25]$. To develop a PDF model of the irradiance consistent with the theory, we make the assumption that small-scale and large-scale irradiance fluctuations are governed by Gamma and Chi-square distribution models, models that both excellently represent small-scale and large-scale turbulence eddy effects, respectively $[\underline{4}, \underline{7}, \underline{13}-\underline{16}]$.

Chi-square distribution is given by the expression [11, 26]:

$$
f_{I_{x}}\left(I_{x}\right)=\frac{1+K}{\Omega_{\mathrm{P}}} \exp \left[-K-\frac{(1+K) I_{x}}{\Omega_{\mathrm{P}}}\right] I_{0}\left(2 \sqrt{\frac{K(1+K)}{\Omega_{\mathrm{P}}} I_{x}}\right), \quad I_{x}>0
$$

where $I_{x}$ is the irradiance at the receiver, $K$ is the ratio of the power of the LOS component to the average power of the scattered component [11], $\Omega_{\mathrm{P}}$ is the total received signal power, while $I_{v}\left({ }^{\circ}\right)$ is the $v$-th-order modified Bessel function of the first kind [2], Eq. 8.431].

Gamma distribution is given by the expression [4]]:

$$
f_{I_{y}}\left(I_{y}\right)=\frac{\alpha\left(\alpha I_{y}\right)^{\alpha-1}}{\Gamma(\alpha)} \exp \left(-\alpha I_{y}\right), \quad I_{y}>0, \quad \alpha>0
$$

where $I_{y}$ is the irradiance at the receiver and $\Gamma(\cdot)$ represents the Gamma function [27, Eq. 8.310]. The parameter $\alpha$ is the atmospheric turbulence parameter that represents the effective numbers of small-scale eddies of the scattering environment. This is the parameter of the atmospheric turbulence which for the propagation of plane waves and zero inner scale can be expressed as $[\underline{4}, \underline{22}]$ :

$$
\alpha=\left\{\exp \left[\frac{0.49 \sigma_{\mathrm{R}}^{2}}{\left(1+1.11 \sigma_{\mathrm{R}}^{12 / 5}\right)^{7 / 6}}\right]-1\right\}^{-1}
$$

where $\sigma_{\mathrm{R}}^{2}$ represents the Rytov variance used to determine the intensity of the optical signal due to atmospheric turbulence, and is defined as:

$$
\sigma_{\mathrm{R}}^{2}=1.23 C_{n}^{2} k^{7 / 6} L^{11 / 6}
$$


The parameter $C_{n}^{2}$ denotes the index of refraction used as a measure of the turbulence strength. For the horizontal propagation path, the parameter $C_{n}^{2}$ is considered constant with mean values from $10^{-17}$ to $10^{-13} \mathrm{~m}^{-2 / 3}$ for channels from weak to strong turbulence, respectively. The parameter $k$ is an optical wave number, defined as $k=2 \pi / \lambda$ with wavelength $\lambda$, while $L$ is the distance between the transmitter and the receiver, i.e. the length of the optical signal propagation.

Using identity $I_{\mathrm{a}}=I_{x} I_{y}$, for atmospheric turbulence model the PDF can be obtained as:

$$
f_{I_{\mathrm{a}}}\left(I_{\mathrm{a}}\right)=\int_{0}^{\infty} f_{I_{x}}\left(I_{\mathrm{a}} \mid I_{y}\right) f_{I_{y}}\left(I_{y}\right) \mathrm{d} I_{y}
$$

By substituting Eqs. (1) and (2) into Eq. (5) and expressing the modified Bessel function of the first kind $I_{v}(\cdot)$ in Eq. (1) in the form of an infinite series [27, Eq. 8.445], as well as by using the solution of the resulting integral given by [27, Eq. 3.478.4], we obtain the closed-form expression for PDF for the Gamma-Chi-square model as follows:

$$
\begin{aligned}
f_{I_{\mathrm{a}}}\left(I_{\mathrm{a}}\right)=\sum_{m=0}^{\infty} & \frac{2 K^{m} \exp (-K)}{\Gamma(\alpha) \Gamma(m+1) m !}\left(\frac{\alpha(1+K)}{\Omega_{\mathrm{P}}}\right)^{\frac{\alpha+m+1}{2}} \\
& \times I_{\mathrm{a}}^{\frac{\alpha+m-1}{2}} K_{\alpha-m-1}\left(2 \sqrt{\frac{\alpha(1+K)}{\Omega_{\mathrm{P}}} I_{\mathrm{a}}}\right)
\end{aligned}
$$

where $K_{v}(\cdot)$ is the $v$-th-order modified Bessel function of the second kind [27, Eq. 8.432].

Based on Eq. (6) mathematical expectations for observed PDF can easily be presented in a form of:

$$
\begin{aligned}
& E\left[I_{\mathrm{a}}\right]=\int_{0}^{\infty} I_{\mathrm{a}} f_{I_{\mathrm{a}}}\left(I_{\mathrm{a}}\right) \mathrm{d} I_{\mathrm{a}}=\Omega_{\mathrm{P}} \\
& E\left[I_{\mathrm{a}}^{2}\right]=\int_{0}^{\infty} I_{\mathrm{a}}^{2} f_{I_{\mathrm{a}}}\left(I_{\mathrm{a}}\right) \mathrm{d} I_{\mathrm{a}}=\Omega_{\mathrm{P}}^{2} \frac{\alpha+1}{\alpha} \frac{K^{2}+4 K+2}{(K+1)^{2}}
\end{aligned}
$$

The scintillation index for the given model now can be presented as:

$$
\mathrm{SI}=\frac{E\left[I_{\mathrm{a}}^{2}\right]}{E^{2}\left[I_{\mathrm{a}}\right]}-1=\frac{\alpha+1}{\alpha} \frac{K^{2}+4 K+2}{(K+1)^{2}}-1
$$

The instantaneous signal-to-noise ratio (SNR) can be derived as follows [26, 27]:

$$
\gamma_{\mathrm{a}}=\frac{\mu_{\mathrm{a}}}{E^{2}\left[I_{\mathrm{a}}\right]} I_{\mathrm{a}}^{2}=\frac{\mu_{\mathrm{a}}}{\Omega_{\mathrm{P}}^{2}} I_{\mathrm{a}}^{2}
$$




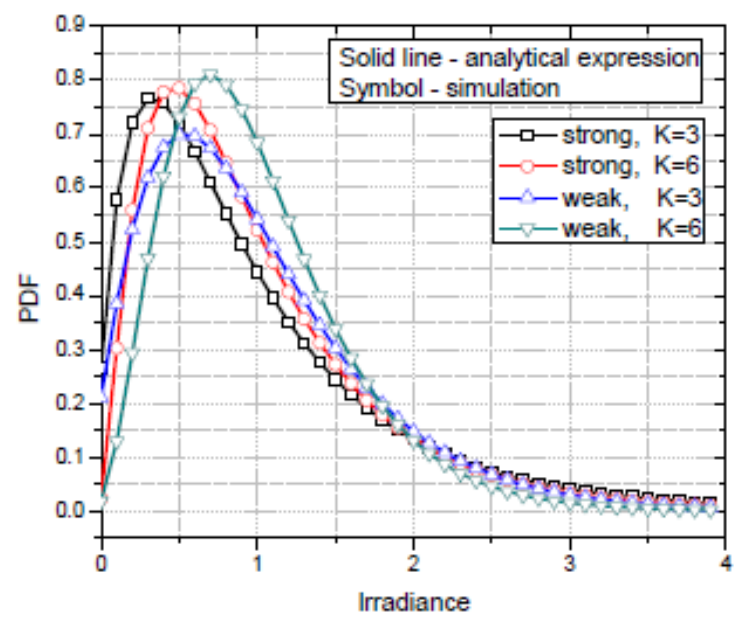

Fig. 1. PDF for the Gamma-Chi-square channel model observed for strong and weak atmospheric turbulence.

where $\mu_{\mathrm{a}}$ represents the average electrical SNR [료, 29]:

$$
\mu_{\mathrm{a}}=\frac{\left(2 P_{\mathrm{T}} R\right)^{2}}{2 \sigma_{\mathrm{N}}^{2}} E^{2}\left[I_{\mathrm{a}}\right]=\frac{\left(2 P_{\mathrm{T}} R \Omega_{\mathrm{P}}\right)^{2}}{2 \sigma_{\mathrm{N}}^{2}}
$$

while $P_{\mathrm{T}}$ denotes the average transmitted optical power, $R$ is the detector responsivity, and $\sigma_{\mathrm{N}}$ is the noise variance.

Using Eqs. (6) and (10), after some mathematical manipulations, the PDF of instantaneous SNR can be obtained:

$$
\begin{aligned}
f_{\gamma_{\mathrm{a}}}\left(\gamma_{\mathrm{a}}\right)=\sum_{m=0}^{\infty} & \frac{K^{m} \exp (-K)}{\Gamma(\alpha) \Gamma(m+1) m !}\left(\frac{\alpha(1+K)}{\sqrt{\mu_{\mathrm{a}}}}\right)^{\frac{\alpha+m+1}{2}} \\
& \times \gamma_{\mathrm{a}}^{\frac{\alpha+m-3}{4}} K_{\alpha-m-1}\left(2 \sqrt{\alpha(1+K) \sqrt{\frac{\gamma_{\mathrm{a}}}{\mu_{\mathrm{a}}}}}\right)
\end{aligned}
$$

Figure 1 shows the PDF curves for newly derived irradiance model when different values of the $K$ factor are observed (strong and weak turbulence).

\subsection{Pointing error model}

Beside the atmospheric turbulence, the pointing error is another effect which can influence the attenuation of the signal in the channel of the FSO system due to the wrong 
pointing of the transmitting laser and the receiving detector. The zero boresight model describes the positioning error which is considered including the optical beam radius at a certain distance from the transmitter, and the circular detector aperture radius and jitter variance $[\underline{19}, \underline{21}, \underline{28}, \underline{29}]$ :

$$
f_{I_{\mathrm{p}}}\left(I_{\mathrm{p}}\right)=\frac{\xi^{2}}{A_{0}^{\xi^{2}}} I_{\mathrm{p}}^{\xi^{2}-1}, \quad 0 \leq I_{\mathrm{p}} \leq A_{0}
$$

The parameter $A_{0}=[\operatorname{erf}(v)]^{2}$ represents the maximal fraction of the collected power, where $\operatorname{erf}(\cdot)$ is the error function [27, Eq. 8.250.1], and $v=\sqrt{\pi} a /\left(\sqrt{2} w_{L}\right)$ with $a$ being the radius of a circular detector aperture, while $w_{L}$ is the optical beam radius at the distance $L$ from transmitter. The parameter $\xi=w_{L_{\mathrm{eq}}} /\left(2 \sigma_{\mathrm{s}}\right)$ is the ratio between the equivalent beam radius at the receiver $w_{L_{\mathrm{eq}}}$, and the pointing error displacement standard deviation (jitter) at the receiver $\sigma_{\mathrm{s}}$, where $w_{L_{\mathrm{eq}}}^{2}=w_{L}^{2} \sqrt{\pi} \operatorname{erf}(v) /\left[2 v \exp \left(-v^{2}\right)\right]$.

Following the same procedure as in [19], by using identity $I=I_{\mathrm{a}} I_{\mathrm{p}}$, for this pointing error model a novel PDF can be obtained as:

$$
f_{I}(I)=\int_{I / A_{0}}^{\infty} f_{I_{\mathrm{p}}}\left(I \mid I_{\mathrm{a}}\right) f_{I_{\mathrm{a}}}\left(I_{\mathrm{a}}\right) \mathrm{d} I_{\mathrm{a}}
$$

By substituting Eqs. (6) and (13) into Eq. (14) and representing the modified Bessel function of the second kind $K_{v}(\cdot)$ by the Meijer G function as [27, Eq. 9.34.3], as well as by using the solution of the resulting integral given by [30, Eq. 07.34.21.0085.01], we obtain the expression for PDF for pointing error for the Gamma-Chi-square model as follows:

$$
f_{I}(I)=\sum_{m=0}^{\infty} \frac{\xi^{2} \alpha(1+K) K^{m} \exp (-K)}{A_{0} \Omega_{\mathrm{P}} \Gamma(\alpha) \Gamma(m+1) m !} G_{1,3}^{3,0}\left[\left.\frac{\alpha(1+K)}{A_{0} \Omega_{\mathrm{P}}} I\right|_{\xi^{2}-1, \alpha-1, m} ^{\xi^{2}}\right]
$$

after applying the relation [르, Eq. 9.31.5] to transform Meijer $\mathrm{G}$ function.

In the Table, the number of terms to be summed in order to achieve accuracy at the desired significant digit is depicted. The calculation was performed for the parameters: $\Omega_{\mathrm{p}}=1, \xi=1.25, A=0.0197921, t=0.1$. As we can see from the Table, the increase in the $K$ factor increases the number of terms to be summed in order to achieve accuracy

$\mathrm{T} \mathrm{a} \mathrm{b} 1 \mathrm{e}$. Terms need to be summed in the expression for cumulative distribution function of PDF in Eq. (15) to achieve accuracy at the 6th significant digit presented.

\begin{tabular}{lcll}
\hline $\begin{array}{l}\text { Atmospheric } \\
\text { turbulence }\end{array}$ & $K$ factor & \\
\cline { 2 - 4 }$K=1$ & $K=3$ & $K=6$ \\
\hline$\alpha=4.02$ (strong) & 9 & 15 & 19 \\
$\alpha=6.90$ (moderate) & 9 & 17 & 21 \\
$\alpha=18.32$ (weak) & 14 & 19 & 28 \\
\hline
\end{tabular}


at the 6th significant digit. For higher values of atmospheric turbulence, a higher number of terms to achieve accuracy at the 6th significant digit is required.

Based on Eq. (15) mathematical expectations of derived PDF can easily be obtained as:

$$
\begin{aligned}
& E[I]=\int_{0}^{\infty} I f_{I}(I) \mathrm{d} I=A_{0} \Omega_{\mathrm{P}} \frac{\xi^{2}}{\xi^{2}+1} \\
& E\left[I^{2}\right]=\int_{0}^{\infty} I^{2} f_{I}(I) \mathrm{d} I=A_{0}^{2} \Omega_{\mathrm{P}}^{2} \frac{\xi^{2}}{\xi^{2}+2} \frac{\alpha+1}{\alpha} \frac{K^{2}+4 K+2}{(K+1)^{2}}
\end{aligned}
$$

while the scintillation index for the given model can be expressed as:

$$
\mathrm{SI}=\frac{E\left[I^{2}\right]}{E^{2}[I]}-1=\frac{\alpha+1}{\alpha} \frac{\left(\xi^{2}+1\right)^{2}}{\xi^{2}+2} \frac{K^{2}+4 K+2}{(K+1)^{2}}-1
$$

The instantaneous SNR can be expressed in this case as:

$$
\gamma=\frac{\mu}{E^{2}[I]} I^{2}=\frac{\mu\left(\xi^{2}+1\right)^{2}}{A_{0}^{2} \Omega_{\mathrm{P}}^{2} \xi^{4}} I^{2}
$$

where $\mu$ represents the average electrical SNR:

$$
\mu=\frac{\left(2 P_{\mathrm{T}} R\right)^{2}}{2 \sigma_{\mathrm{N}}^{2}} E^{2}[I]=\frac{\left(2 P_{\mathrm{T}} R A_{0} \Omega_{\mathrm{P}} \xi^{2}\right)^{2}}{2 \sigma_{\mathrm{N}}^{2}\left(\xi^{2}+1\right)^{2}}
$$

Using Eq. (15) and Eq. (19), after some mathematical manipulations, the PDF of instantaneous SNR can be obtained:

$$
f_{\gamma}(\gamma)=\sum_{m=0}^{\infty} \frac{\xi^{2} K^{m} \exp (-K)}{2 \Gamma(\alpha) \Gamma(m+1) m ! \gamma} G_{1,3}^{3,0}\left[\left.\frac{\xi^{2} \alpha(1+K)}{\xi^{2}+1} \sqrt{\frac{\gamma}{\mu}}\right|_{\xi^{2}, \alpha, m+1} ^{\xi^{2}+1}\right]
$$

\section{Performance analysis}

The ABER which depends on the SNR at the receiver for an optical signal transmitted by FSO system with the OOK modulation scheme can be expressed as [31, 32]

$$
P_{\mathrm{e}}=\int_{0}^{\infty} \frac{1}{2} \operatorname{erfc}\left(\frac{\sqrt{\gamma}}{2}\right) f_{\gamma}(\gamma) \mathrm{d} \gamma
$$

where $\operatorname{erfc}(\cdot)$ is a complementary error function [27, Eq. 8.250.4], and $f_{\gamma}(\gamma)$ represents PDF of instantaneous SNR of the received signal. 


\subsection{Atmospheric turbulence model}

Substituting Eq. (12) into Eq. (22), and then representing $\operatorname{erfc}(\cdot)$ by the Meijer G function as [33, Eq. 06.27.26.0003.01] and the modified Bessel function of the second kind $K_{v}(\cdot)$ by the Meijer G function as [27, Eq. 9.34.3], we get the resulting integral. By using the solution of the resulting integral given by [30, Eq. 07.34.21.0013.01], we derive the ABER expression for atmospheric turbulence Gamma-Chi-square channel model as follows:

$$
P_{\mathrm{e}}\left(\mu_{\mathrm{a}}\right)=\sum_{m=0}^{\infty} \frac{2^{\alpha+m-2} K^{m} \exp (-K)}{\pi^{3 / 2} \Gamma(\alpha) \Gamma(m+1) m !} G_{2,5}^{4,2}\left[\left.\frac{\alpha^{2}(1+K)^{2}}{4 \mu_{\mathrm{a}}}\right|_{\frac{\alpha}{2}, \frac{\alpha+1}{2}, \frac{m+1}{2}, \frac{m+2}{2}, 0} ^{1, \frac{1}{2}}\right]
$$

Based on the definition of the average electrical SNR in Eq. (11), the ABER can be represented as a function of the average optical power at the transmission $P_{\mathrm{T}}$, as:

$$
\begin{aligned}
P_{\mathrm{e}}\left(P_{\mathrm{T}}\right)=\sum_{m=0}^{\infty} & \frac{2^{\alpha+m-2} K^{m} \exp (-K)}{\pi^{3 / 2} \Gamma(\alpha) \Gamma(m+1) m !} \\
& \times G_{2,5}^{4,2}\left[\left.\frac{\alpha^{2}(1+K)^{2} \sigma_{\mathrm{N}}^{2}}{8 P_{\mathrm{T}}^{2} R^{2} \Omega_{\mathrm{P}}^{2}}\right|_{\left.\frac{\alpha}{2}, \frac{\alpha+1}{2}, \frac{m+1}{2}, \frac{m+2}{2}, 0\right]} ^{1, \frac{1}{2}}\right.
\end{aligned}
$$

\subsection{Pointing error model}

By substituting Eq. (21) into Eq. (22), and representing erfc( $\cdot$ ) by the Meijer G function as [33, Eq. 06.27.26.0003.01], as well as by using the solution of the resulting integral given by [30, Eq. 07.34.21.0013.01], we derive the ABER expression for pointing error Gamma-Chi-square channel model as follows:

$$
\begin{aligned}
P_{\mathrm{e}}(\mu)=\sum_{m=0}^{\infty} & \frac{2^{\alpha+m-3} \xi^{2} K^{m} \exp (-K)}{\pi^{3 / 2} \Gamma(\alpha) \Gamma(m+1) m !} \\
& \times G_{3,6}^{5,2}\left[\left.\frac{\xi^{4} \alpha^{2}(1+K)^{2}}{4 \mu\left(\xi^{2}+1\right)^{2}}\right|_{\left.\frac{\xi^{2}}{2}, \frac{\alpha}{2}, \frac{\alpha+1}{2}, \frac{m+1}{2}, \frac{m+2}{2}, 0\right]} ^{1, \frac{\xi^{2}+2}{2}}\right.
\end{aligned}
$$

Based on the definition of the average electrical SNR in Eq. (20), the ABER can be represented as a function of the average optical power at the transmission $P_{\mathrm{T}}$, as: 


$$
\begin{aligned}
P_{\mathrm{e}}\left(P_{\mathrm{T}}\right)=\sum_{m=0}^{\infty} & \frac{2^{\alpha+m-3} \xi^{2} K^{m} \exp (-K)}{\pi^{3 / 2} \Gamma(\alpha) \Gamma(m+1) m !} \\
& \times G_{3,6}^{5,2}\left[\left.\frac{\alpha^{2}(1+K)^{2} \sigma_{\mathrm{N}}^{2}}{8 P_{\mathrm{T}}^{2} R^{2} A_{0}^{2} \Omega_{\mathrm{P}}^{2}}\right|^{1, \frac{1}{2}, \frac{\xi^{2}+2}{2}}, \frac{\alpha}{2}, \frac{\alpha+1}{2}, \frac{m+1}{2}, \frac{m+2}{2}, 0\right]
\end{aligned}
$$

\section{Numerical results}

For the numerical calculation, the FSO system was observed at the wavelength $\lambda=$ $=1550 \mathrm{~nm}$ and at the distance between the transmitter and the receiver $L=1 \mathrm{~km}$. Three types of atmospheric turbulence were considered: weak, moderate and strong, with indexes of reflection $C_{n}^{2}=6 \times 10^{-15} \mathrm{~m}^{-2 / 3}, C_{n}^{2}=2 \times 10^{-14} \mathrm{~m}^{-2 / 3}$ and $C_{n}^{2}=1.2 \times 10^{-13} \mathrm{~m}^{-2 / 3}$, respectively. Total received signal power is $\Omega_{\mathrm{P}}=1$, while detector responsivity $R=1 \mathrm{~A} / \mathrm{W}$ and noise variance $\sigma_{\mathrm{N}}=10^{-7} \mathrm{~A} / \mathrm{Hz}$. The radius of a circular detector aperture $a=0.05 \mathrm{~m}$, the optical beam radius at the distance $L$ from transmitter $w_{L}=0.5 \mathrm{~m}$, pointing error displacement standard deviation (jitter) at the receiver $\sigma_{\mathrm{s}}=0.2 \mathrm{~m}$ are considered.

In Figs. 2 and 3 the ABER behavior of the FSO channel modelled with the Gamma -Chi-square distribution is shown, as a function of the average electrical SNR in the case of atmospheric turbulence and a pointing error model, respectively. The ABER for different strength levels of atmospheric turbulence, different values of the $K$ factor and OOK modulation scheme expressed by Eqs. (23) and (25) are graphically presented. From Fig. 2 and Fig. 3 it can be seen that the increase of the average electrical SNR leads

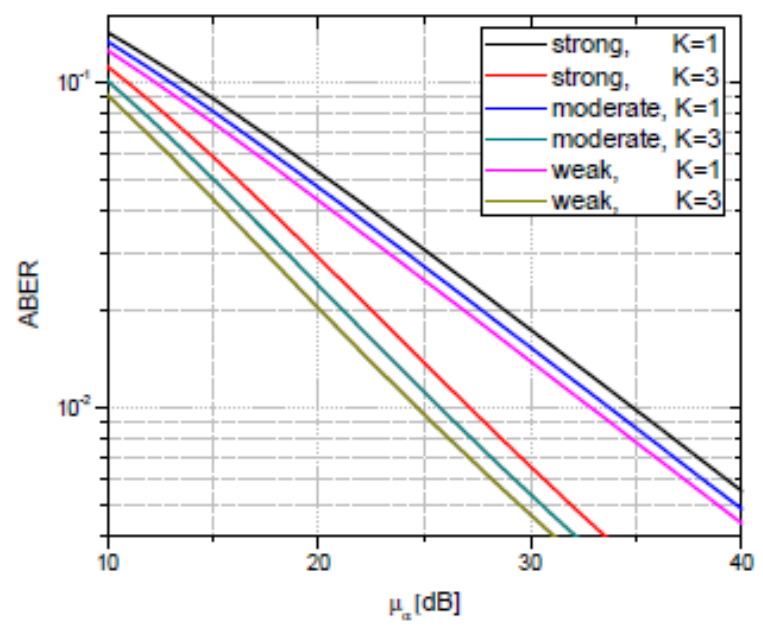

Fig. 2. ABER for the Gamma-Chi-square atmospheric turbulence channel model as a function of the average electrical SNR. 


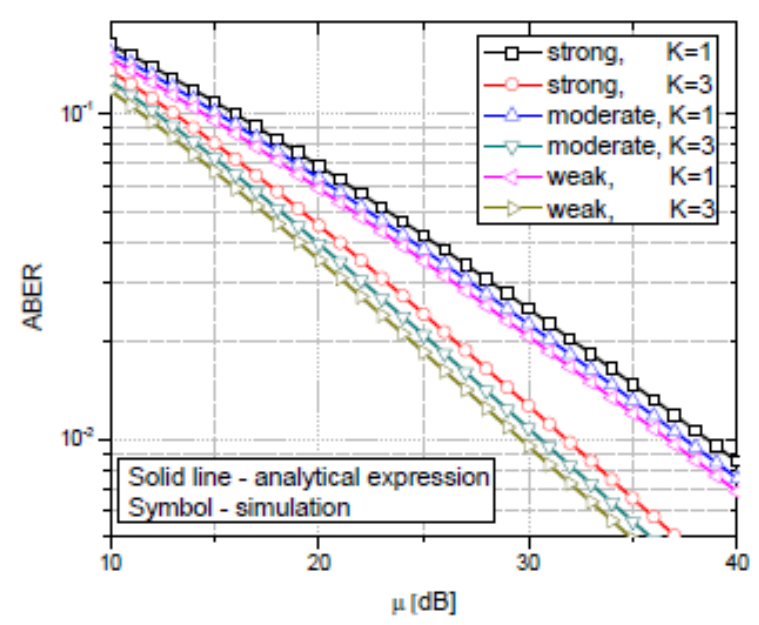

Fig. 3. ABER for the Gamma-Chi-square pointing error channel model as a function of the average electrical SNR.

to a decrease in ABER. Also, the ABER change is approximately linear in both cases. As expected, for stronger turbulence and lower values of the $K$ factor, the higher ABER values are obtained. In both the atmospheric turbulence and the pointing error model, the ABER decreases significantly faster for higher values of the $K$ factor than for those lower. With the increase of the SNR, the dominant ABER differences occur for cases where $K=1$ and $K=3$, while for lower values of the SNR, this difference is much smaller. The FSO system has better performance, i.e. ABER is lower for the same fit conditions, when signal attenuation is only affected by atmospheric turbulence, which is the case with the pointing error impact.

In order to validate obtained results, we have carried out Monte Carlo simulations for observed PDF and ABER scenarios and showed that they excellently fit the values obtained from analytical expressions. As it can be seen in Figs. 1 and 3, PDF and ABER values obtained by using derived infinite-series expressions and their corresponding values obtained by Monte Carlo simulations excellently concede.

Based on the closed-form analytical expression obtained for ABER for the pointing error model, Eq. (26), the graphs of ABER change depending on the function of the average optical power at the transmission $P_{\mathrm{T}}$, are presented. The results were obtained for the case of moderate atmospheric turbulence and for the value of $K$ factor $K=2$.

Figure 4 shows the ABER behavior of Gamma-Chi-square channel model, with applied OOK modulation scheme as a function of the average optical power at the transmission $P_{\mathrm{T}}$, for different values of the normalized standard jitter deviation at the receiver $\sigma_{\mathrm{s}}$. From Fig. 4 it can be seen that the ABER decreases linearly as the $P_{\mathrm{T}}$ increases. Also, for higher values of the normalized standard jitter deviation $\sigma_{\mathrm{s}}$ the higher ABER values are obtained. Wrong transmitter and receiver positioning is manifested 


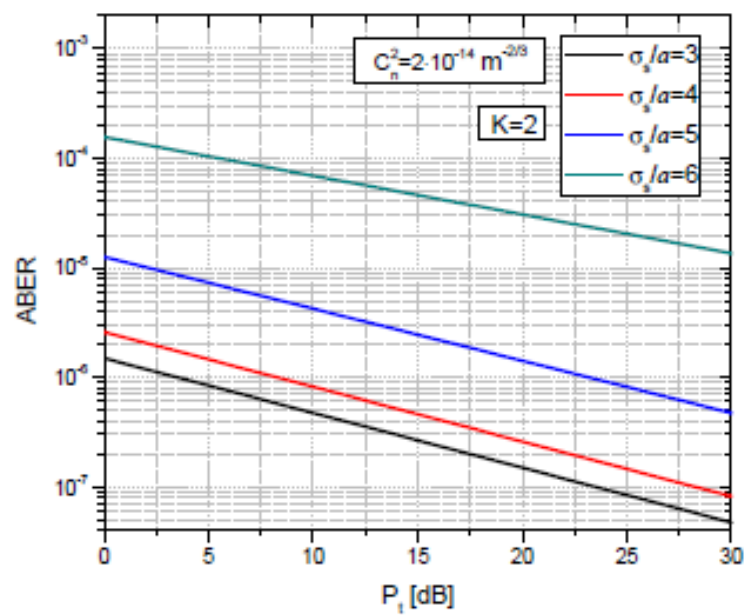

Fig. 4. ABER for the Gamma-Chi-square pointing error channel model for different values of the normalized standard jitter deviation.

by higher values of standard jitter deviation $\sigma_{\mathrm{s}}$, which leads to a deterioration in system performance.

Figure 5 shows ABER behavior of Gamma-Chi-square channel model, with applied OOK modulation scheme as a function of the average optical power at the transmission $P_{\mathrm{T}}$, for different values of the normalized optical beam radius $w_{L}$ at the distance $L$ from the transmitter. Since the optical beam radius $w_{L}$ depends on the distance $L$ from the transmitter, it is clear that higher values of this parameter greatly affect the dete-

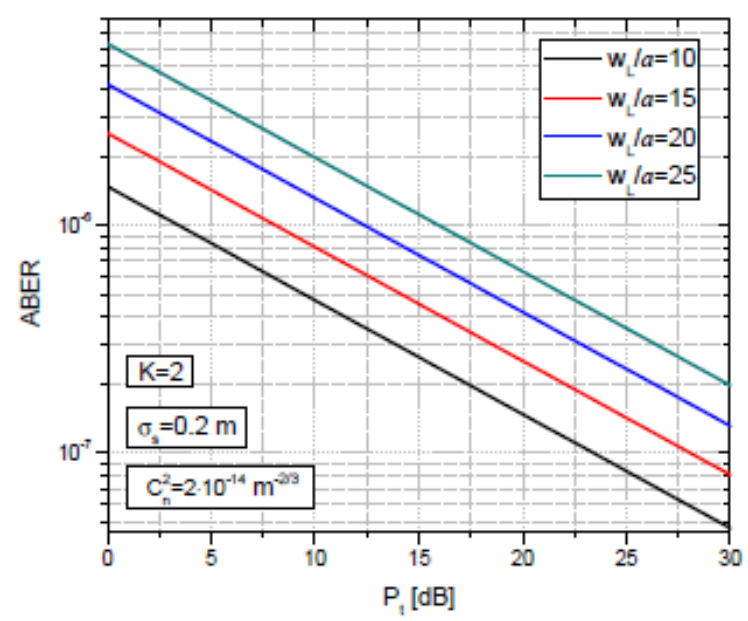

Fig. 5. ABER for the Gamma-Chi-square pointing error channel model for different values of the normalized optical beam radius. 
rioration of ABER, as well as the system performance. Figure 5 indicates that the ABER decreases linearly as the $P_{\mathrm{T}}$ increases, as well as ABER taking higher values with increasing of the normalized optical beam radius $w_{L}$.

\section{Conclusions}

In this paper, we have introduced a novel general Gamma-Chi-square FSO channel model for modeling irradiance fluctuations in a wide range of turbulence conditions. Further, boresight pointing error conditions have been taken into account, and capitalizing on newly proposed Gamma-Chi-square model, closed-form PDF expression have been introduced for modeling such FSO transmission scenario. Subsequently, we have carried out the performance analysis over Gamma-Chi-square channels assuming IM/DD with OOK, and have derived closed-form ABER expressions for both transmission cases, with and without pointing error effects. The obtained numerical results are graphically presented in order to analyse the impact of different levels of turbulence, as well as other relevant parameters on the quality of signal transmission.

\section{References}

[1] ChuRnside J.H., Clifford S.F., Log-normal Rician probability-density function of optical scintillations in the turbulent atmosphere, Journal of the Optical Society of America A 4(10), 1987, pp. 1923 -1930, DOI: 10.1364/JOSAA.4.001923.

[2] Jakeman E., Pusey P., Significance of $K$ distributions in scattering experiments, Physical Review Letters 40(9), 1978, pp. 546-550, DOI: 10.1103/PhysRevLett.40.546.

[3] Andrews L., Phillips R.L., Mathematical genesis of the I-K distribution for random optical fields, Journal of the Optical Society of America A 3(11), 1986, pp. 1912-1919, DOI: 10.1364/JOSAA.3.001912.

[4] Al-Habash A., Andrews L.C., Phillips R.L., Mathematical model for the irradiance probability density function of a laser beam propagating through turbulent media, Optical Engineering 40(8), 2001, pp. 1554-1562, DOI: 10.1117/1.1386641.

[5] Jurado-Navas A., Garrido-Balsells J.M., Paris J.F., Puerta-Notario A., A unifying statistical model for atmospheric optical scintillation, arXiv preprint arXiv:1102.1915, 2011, DOI: 10.5772/ 25097.

[6] Chatzidiamantis N.D., Sandalidis H.G., Karagiannidis G.K., Kotsopoulos S.A., Matthaiou M., New results on turbulence modeling for free-space optical systems, Proceedings of 2010 17th IEEE International Conference on Telecommunications (ICT), April 4-7, 2010, Doha, Qatar, pp. 487-492, DOI: $10.1109 /$ ICTEL.2010.5478872.

[7] Kashani M.A., Uysal M., Kavehrad M., A novel statistical channel model for turbulence-induced fading in free-space optical systems, Journal of Lightwave Technology 33(11), 2015, pp. 2303-2312, DOI: $10.1109 /$ JLT.2015.2410695.

[8] Bithas P.S., Kanatas A.G., Da Costa D.B., Upadhyay P.K., Dias U.S., On the double-generalized Gamma statistics and their application to the performance analysis of V2V communications, IEEE Transactions on Communications 66(1), 2018, pp. 448-460, DOI: 10.1109/TCOMM.2017.2757466.

[9] ChuRnside J.H., Frehlich R.G., Experimental evaluation of log-normally modulated Rician and IK models of optical scintillation in the atmosphere, Journal of the Optical Society of America A 6(11), 1989, pp. 1760-1766, DOI: 10.1364/JOSAA.6.001760.

[10] Yang F., Cheng J., Coherent free-space optical communications in lognormal-Rician turbulence, IEEE Communications Letters 16(11), 2012, pp. 1872-1875, DOI: 10.1109/LCOMM.2012.100812. 121341. 
[11] Belmonte A., Kahn J.M., Performance of synchronous optical receivers using atmospheric compensation techniques, Optics Express 16(18), 2008, pp. 14151-14162, DOI: 10.1364/OE.16.014151.

[12] Zhou H., Xie W., Zhang L., Bai Y., Wei W., Dong Y., Performance analysis of FSO coherent BPSK systems over Rician turbulence channel with pointing errors, Optics Express 27(19), 2019, pp. 27062-27075, DOI: 10.1364/OE.27.027062.

[13] Aghajanzadeh S.M., Uysal M., Diversity-multiplexing trade-off in coherent free-space optical systems with multiple receivers, Journal of Optical Communications and Networking 2(12), 2010, pp. 1087-1094, DOI: 10.1364/JOCN.2.001087.

[14] Panić S., MilošEvić H., PruinčEvić B., Performance analysis of FSO transmission of double watermarked image over the double Rician turbulence channel, Proceedings of First West Asian Colloquium on Optical Wireless Communications (WACOWC), April 25, 2018, Isfehan, Iran.

[15] Panić S., Milošević H., Vasić S, Milenković V., Dynamical characteristics of the FSO transmission capacity in the presence of Rician turbulence, Proceedings of 2018 International Conference on Information and Communications Technology (ICOIACT), March 6-7, 2018, Jogyakarta, Indonesia.

[16] Prlinčević B.P., Panić S.R., Spalević P.C., Mišić M.A., Amniesi A., Stanojević V., On the transmission of double watermarked image over Rician FSO channel, Elektronika ir Elektrotechnika 22(3), 2016, pp. 83-88, DOI: 10.5755/j01.eie.22.3.15320.

[17] SaXena P., Mathur A., Bhatnagar M.R., BER performance of an optically pre-amplified FSO system under turbulence and pointing errors with ASE noise, Journal of Optical Communications and Networking 9(6), 2017, pp. 498-510, DOI: 10.1364/jocn.9.000498.

[18] Elsayed E.E., Yousir B.B., Performance enhancement of the average spectral efficiency using an aperture averaging and spatial-coherence diversity based on the modified-PPM modulation for MISO FSO links, Optics Communications 463, 2020, article 125463, DOI: 10.1016/j.optcom.2020.125463.

[19] Smilić M., Perić Z., Milić D., Marković A., Savić M., Influence of zero and non-zero boresight pointing errors on bit-error rate free-space optic transmission over Málaga atmospheric channel, Optica Applicata 48(2), 2018, pp. 179-189, DOI: 10.5277/oa180202.

[20] Ansari I.S., Alouini M.S., Cheng J., Ergodic capacity analysis of free-space optical links with nonzero boresight pointing errors, IEEE Transactions on Wireless Communications 14(8), 2015, pp. 4248-4264, DOI: 10.1109/TWC.2015.2418285.

[21] Sandalidis H.G., TsifTsis T.A., Karagiannidis G.K., Uysal M., BER performance of FSO links over strong atmospheric turbulence channels with pointing errors, IEEE Communications Letters 12(1), 2008, pp. 44-46, DOI: 10.1109/LCOMM.2008.071408.

[22] Sandalidis H.G., Tsiftsis T.A., KaragianNidis G.K., Optical wireless communications with heterodyne detection over turbulence channels with pointing errors, Journal of Lightwave Technology 27(20), 2009, pp. 4440-4445, DOI: 10.1109/JLT.2009.2024169.

[23] Wang Z., Zhong W.-D., Fu S., Lin C., Performance comparison of different modulation formats over free-space optical (FSO) turbulence links with space diversity reception technique, IEEE Photonics Journal 1(6), 2009, pp. 277-285, DOI: 10.1109/JPHOT.2009.2039015.

[24] Tsiftsis T.A., Sandalidis H.G., Karagiannidis G.K., Uysal M., Optical wireless links with spatial diversity over strong atmospheric turbulence channels, IEEE Transactions on Wireless Communications 8(2), 2009, pp. 951-957, DOI: 10.1109/TWC.2009.071318.

[25] Milošević N.D., Petković M.I., Duordjević G.T., Average BER of SIM-DPSK FSO system with multiple receivers over $M$-distributed atmospheric channel with pointing errors, IEEE Photonics Journal 9(4), 2017, article 6601210, DOI: 10.1109/JPHOT.2017.2710320.

[26] StüBer G.L., Principles of Mobile Communication, 2nd Edition, Kluwer Academic Publishers, 2002.

[27] Gradshteyn I.S., RyzhiK I.M., Table of Integrals, Series, and Products, 7th Ed., Elsevier Academic Press, 2007.

[28] Djordjević G.T., Petković M.I., Spasić M., Antić D.S., Outage capacity of FSO link with pointing errors and link blockage, Optics Express 24(1), 2016, pp. 219-230, DOI: 10.1364/oe.24.000219.

[29] Farid A.A., Hranilović S., Outage capacity optimization for free-space optical links with pointing errors, Journal of Lightwave Technology 25(7), 2007, pp. 1702-1710, DOI: 10.1109/JLT.2007.899174. 
[30] The Wolfarm Functions Site: MeijerG functions, http://functions.wolfram.com/PDF/MeijerG.pdf (accessed May 2020).

[31] SAwhiL P.B., Effect of atmospheric turbulence and pointing error on OOK in free space optics, International Journal of Engineering Trends and Technology (IJETT) 59(3), 2018, pp. 122-126, DOI: $10.14445 / 22315381 /$ IJETT-V59P221.

[32] Zhang H., Li H., Hao C., Performance analysis for BPSK, DPSK and OOK-based FSO system in atmospheric turbulence conditions, International Journal of Simulation - Systems, Science \& Technology 17(36), 2016, article 37.1, DOI: 10.5013/ IJSSST.a.17.36.37.

[33] The Wolfarm Functions Site: Erfc functions, http://functions.wolfram.com/PDF/Erfc.pdf (accessed May 2020).

Received May 9, 2020

in revised form August 24, 2020 